\title{
Criteria for Synchronization of Coupled Chaotic External-Cavity Semiconductor Lasers
}

\author{
José Revuelta, Claudio R. Mirasso, Pere Colet, and Luís Pesquera
}

\begin{abstract}
In this letter, we study the synchronization of two unidirectionally coupled single-mode external-cavity semiconductor lasers, that operate in a chaotic regime. A simple theory is developed to obtain synchronization conditions and to analyze the effects of the detuning between the two lasers. We find that numerical simulations are in good agreement with the theory.
\end{abstract}

Index Terms-Chaos, feedback lasers, injection locked oscillators, semiconductor lasers, synchronization.

$\mathbf{T}$ HE IDEA OF enhancing the privacy in transmitted data by using devices (emitters and receivers) operating in a chaotic regime has attracted the attention of many researchers in the last years. Different chaotic synchronization schemes and their applications to encoded communications have been proposed using electronic circuits [1], solid state lasers [2], fiber ring lasers [3], semiconductor lasers [4]-[13], and microchip lasers [14].

Experimental studies have already shown the feasibility of synchronizing the so-called hyperchaos in optical systems [3], [7]-[10]. Of special interest are laser diode (LD) systems with optical feedback. Recent experimental results [8], [10] have shown that synchronization can be achieved in a rather large parameter range.

Our aim in this letter is to develop a theory that allows us to predict the characteristics of the synchronization between two unidirectionally coupled external-cavity chaotic LD. In particular, we determine the existence of an optimal value for the coupling and the parameter region where synchronization is possible. We consider that the emitter [master laser (ML)] and the receiver [slave laser (SL)] can have different emission wavelengths and photon lifetimes. A rate equation model is used for the complex slowly varying amplitude of the electrical field $E_{\mathrm{m}, \mathrm{s}}$ and the minority carriers inside the cavity $N_{\mathrm{m}, \mathrm{s}}$, where indexes $\mathrm{m}$ and $\mathrm{s}$ label the ML and SL, respectively. The slowly varying amplitude $E_{\mathrm{m}, \mathrm{s}}$ is defined as $\epsilon_{\mathrm{m}, \mathrm{s}}(t)=E_{\mathrm{m}, \mathrm{s}}(t) e^{j \Omega t}+$ c.c., where $\epsilon_{\mathrm{m}, \mathrm{s}}(t)$ is the electrical field, $j$ the imaginary unit and $\Omega=\left(\omega_{\mathrm{m}}+\omega_{\mathrm{s}}\right) / 2$, being $\omega_{\mathrm{m}, \mathrm{s}}$ the optical laser frequencies

Manuscript received August 8, 2001; revised October 04, 2001. This work was supported by the DGES under Project PB97-0141-C02-01/02, by the MCyT under Project BFM2000-1108, by the CICYT under Project TIC99-0645), and by the Spain and European Commission under Project OCCULT, IST-200029683.

J. Revuelta and L. Pesquera are with the Instituto de Física de Cantabria, CSIC-UC, Av. Los Castros s/n E-39005 Santander, Spain.

C. R. Mirasso is with the Departament de Física, Universitat de les Illes Balears, E-07071 Palma de Mallorca, Spain (e-mail: claudio@imedea.uib.es).

P. Colet is with the Instituto Mediterráneo de Estudios Avanzados, CSIC-UIB,

Campus UIB, E-07071 Palma de Mallorca, Spain.

Publisher Item Identifier S 1041-1135(02)00275-6. under continuous-wave (CW) operation. In terms of intensity and phase $\left(E_{\mathrm{m}, \mathrm{s}}(t)=\sqrt{I_{\mathrm{m}, \mathrm{s}}(t)} e^{j \psi_{\mathrm{m}, \mathrm{s}}(t)}\right)$ the equation read

$$
\begin{aligned}
\dot{I}_{\mathrm{m}, \mathrm{s}}(t)= & \left(G_{\mathrm{m}, \mathrm{s}}(t)-\gamma_{\mathrm{m}, \mathrm{s}}\right) I_{\mathrm{m}, \mathrm{s}}(t) \\
& +2 \kappa_{\mathrm{m}, \mathrm{s}} \sqrt{I_{\mathrm{m}, \mathrm{s}}\left(t-\tau_{\mathrm{m}, \mathrm{s}}\right) I_{\mathrm{m}, \mathrm{s}}(t)} \cos \xi_{\mathrm{m}, \mathrm{s}} \\
& +2 \kappa_{c} \sqrt{I_{\mathrm{m}}(t) I_{\mathrm{s}}(t)} \cos \left(\psi_{\mathrm{m}}-\psi_{\mathrm{s}}\right) \\
\dot{\psi}_{\mathrm{m}, \mathrm{s}}= & \pm \frac{\Delta \omega}{2}+\frac{\alpha}{2}\left(G_{\mathrm{m}, \mathrm{s}}(t)-\gamma_{\mathrm{m}, \mathrm{s}}\right) \\
& -\kappa_{\mathrm{m}, \mathrm{s}} \sqrt{\frac{I_{\mathrm{m}, \mathrm{s}}\left(t-\tau_{\mathrm{m}, \mathrm{s}}\right)}{I_{\mathrm{m}, \mathrm{s}}}} \sin \xi_{\mathrm{m}, \mathrm{s}} \\
& +\kappa_{c} \sqrt{\frac{I_{\mathrm{m}}(t)}{I_{\mathrm{s}}(t)} \sin \left(\psi_{\mathrm{m}}-\psi_{\mathrm{s}}\right)} \\
\dot{N}_{\mathrm{m}, \mathrm{s}}(t)= & \frac{J}{e}-\gamma_{n} N_{\mathrm{m}, \mathrm{s}}(t)-G_{\mathrm{m}, \mathrm{s}}(t) I_{\mathrm{m}, \mathrm{s}}(t) \\
G_{\mathrm{m}, \mathrm{s}}(t)= & \frac{g\left(N_{\mathrm{m}, \mathrm{s}}(t)-N_{o}\right)}{\left(1+s I_{\mathrm{m}, \mathrm{s}}(t)\right)}
\end{aligned}
$$

where $\xi_{\mathrm{m}, \mathrm{s}}=\psi_{\mathrm{m}, \mathrm{s}}(t)-\psi_{\mathrm{m}, \mathrm{s}}(t-\tau)+\Omega \tau_{\mathrm{m}, \mathrm{s}}$ and the dot stands for the time derivative. We have taken for simplicity, the propagation time of the coupling signal from the ML to the SL, $\tau_{c}=0^{1}$. The term containing $\kappa_{c}$ in (1), which takes into account the injected signal coming from the ML is only present in the SL. We consider the ML and SL to be very similar to each other, such that some of the internal parameters are taken identical $g=1.5 \times 10^{-8} \mathrm{ps}^{-1}$ is the gain parameter, $s=5 \times 10^{-7}$ is the gain saturation coefficient, $\alpha=5$ is the linewidth enhancement factor, $e=1.602 \times 10^{-19} \mathrm{C}$ is the electron charge, $\gamma_{n}=0.5 \mathrm{~ns}^{-1}$ is the inverse carrier lifetime and $N_{o}=1.5 \times 10^{8}$ is the carrier number at transparency. The ML cavity losses $\gamma_{\mathrm{m}}=0.5 \mathrm{ps}^{-1}$ is fixed, while $\gamma_{\mathrm{s}}$ will be considered variable. The threshold current for the ML is $J_{\mathrm{th}} \sim 17.3 \mathrm{~mA}$ and the bias current $J=2 J_{\mathrm{th}}$ for both lasers. $\kappa_{\mathrm{m}, \mathrm{s}}$ is the feedback coefficient, $\tau_{\mathrm{m}, \mathrm{s}}$ is the external cavity round-trip time, and $\Delta \omega=\omega_{\mathrm{m}}-\omega_{\mathrm{s}}$ is the detuning. The ML emission wavelength is $\lambda_{\mathrm{m}}=1.55 \mu \mathrm{m}$, while the SLs one $\lambda_{\mathrm{s}}$ will be varied. In what follows, we consider the same feedback coupling coefficients $\kappa_{\mathrm{s}}=\kappa_{\mathrm{m}}=\kappa^{f}$ and delay time $\tau_{\mathrm{m}}=\tau_{\mathrm{s}}=\tau=1 \mathrm{~ns}$.

Naively, one would like to have a perfect synchronization between the two lasers, this is $I_{\mathrm{s}}=I_{\mathrm{m}}$ and $N_{\mathrm{s}}=N_{\mathrm{m}}$. However, even in the case of identical internal parameters, this cannot be easily achieved, due to the presence of an injected field in the SL which is absent in the ML. However, and for purposes of [13]

${ }^{1}$ For Discussions on the Role of $\tau_{c}$ in the Synchronization See [6], [9]-[11], 
encoded communications, the scheme can work without major inconvenience if one has

$$
I_{\mathrm{s}}(t)=a I_{\mathrm{m}}(t),
$$

with $a$ being constant in time. This condition implies $\dot{I}_{\mathrm{s}}(t)=$ $a \dot{I}_{\mathrm{m}}(t)$ which can only be verified by (1) imposing

$$
\Delta \phi \equiv \psi_{\mathrm{m}}(t)-\psi_{\mathrm{s}}(t)=\text { constant } .
$$

Physically, this means that the frequency of the SL locks to the frequency of the ML. Also for the carriers, we impose $N_{\mathrm{s}}=$ $N_{\mathrm{m}}+\Delta N$ with $\Delta N$ constant in time. As the delay is the same for both lasers, (6) implies $\xi_{\mathrm{s}}=\xi_{\mathrm{m}}$ in (1) and (2). It will be shown later that under these circumstances the synchronization will become independent of the feedback.

From (1), (2) and defining $\Delta G=G_{\mathrm{s}}-G_{\mathrm{m}}$ and $\Delta \gamma=\gamma_{\mathrm{s}}-\gamma_{\mathrm{m}}$ we have

$$
\begin{aligned}
\Delta \gamma-\Delta G & =\frac{2 \kappa_{c}}{\sqrt{a}} \cos \Delta \phi \\
\frac{\alpha}{2}(\Delta \gamma-\Delta G)+\Delta \omega & =\frac{\kappa_{c}}{\sqrt{a}} \sin \Delta \phi .
\end{aligned}
$$

From these equations, we can predict the value of $\Delta \phi$

$$
\Delta \phi=\arctan (\alpha)+\arcsin \left(\frac{\Delta \omega}{\kappa_{c}} \sqrt{\frac{a}{1+\alpha^{2}}}\right)
$$

where $a$ can be calculated by combining (3), (4), and (5)

$$
a=\frac{G_{\mathrm{m}}}{G_{\mathrm{m}}+\Delta G}\left(1-\frac{\Delta G}{G_{\mathrm{m}}} \frac{\gamma_{n}}{\left(g+s \gamma_{n}\right) I_{\mathrm{m}}}\right) \approx \frac{\gamma_{\mathrm{m}}}{\gamma_{\mathrm{m}}+\Delta G}
$$

where we have used $G_{\mathrm{m}} \approx \gamma_{\mathrm{m}}$ and $\gamma_{n} \Delta G /\left[\left(g+s \gamma_{n}\right) I_{\mathrm{m}} G_{\mathrm{m}}\right] \ll$ 1. Using (7)-(10), $\Delta G$ is obtained see (11) at the bottom of the page. This expression of $\Delta G$ corresponds to the root that can be stable in the case of injection locking of lasers in the CW regime [15]. Therefore, using (9)-(11), we are able to obtain the characteristics of the SL output from those of the ML.

A closer look to (11) reveals the fact that a minimum value for the coupling coefficient $\kappa_{c}$, below which synchronization is not possible can be obtained. As $\Delta G$ is a real number, the square root of (11) has to be greater or equal than zero, i.e.,

$$
\kappa_{c}^{\min }=|\Delta \omega| \sqrt{\frac{\gamma_{\mathrm{m}}}{\left(\gamma_{\mathrm{m}}+\Delta \gamma\right)\left(1+\alpha^{2}\right)}}
$$

where we have assumed that $\Delta \omega \ll \gamma_{\mathrm{m}}$. This condition corresponds to the locking regime for the CW operation.

An optimum value for $\kappa_{c}\left(\kappa_{c}^{\text {id }}\right)$ for which both optical intensities are identical, i.e., the value for which $\Delta G=\Delta N=0$ and $a=1$ can be obtained from (7) and (8)

$$
\kappa_{c}^{\mathrm{id}}=\sqrt{\left(\frac{\alpha \Delta \gamma}{2}+\Delta \omega\right)^{2}+\left(\frac{\Delta \gamma}{2}\right)^{2}}
$$

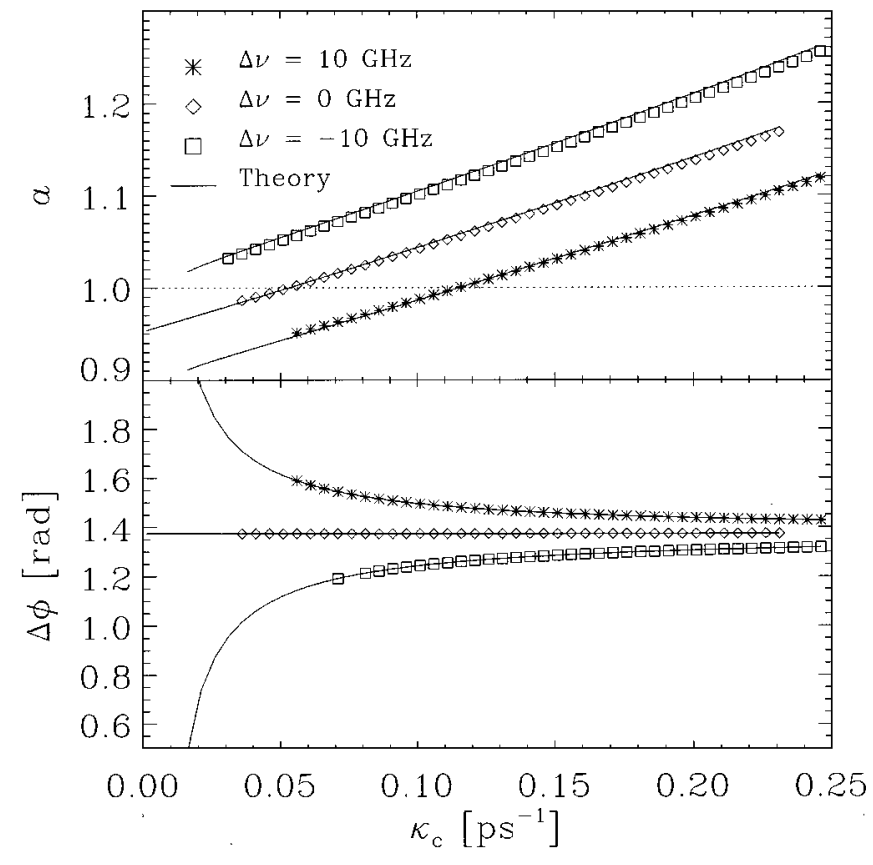

Fig. 1. Values of $a$ and $\Delta \phi$ as function of the coupling constant for $\Delta \gamma=$ $0.02 \mathrm{ps}^{-1}, \kappa^{f}=2.04 \times 10^{-2} \mathrm{ps}^{-1}$ and $\alpha=5$. The solid lines correspond to the predictions given by (9) and (10). The symbols correspond to the results obtained from numerical integration of (1) and (4).

The existence of an optimal coupling comes from the fact that the two lasers are different because the SL has an injected signal. For couplings smaller than the optimum, we obtain $a<1$, while for couplings larger than $\kappa_{c}^{\text {id }} a>1$. Notice that $\kappa_{c}^{\text {id }} \geq \kappa_{c}^{\text {min }}$. Thus, from (12) and (13), we obtain that the optimal synchronization is only possible if

$$
\Delta \omega \geq \Delta \omega_{c}=-\frac{\Delta \gamma\left(1+\alpha^{2}\right)}{2 \alpha} .
$$

Note that the analytic results for the synchronized solution are independent of the feedback and consequently of the kind of dynamics. In what follows, we compare our theoretical results with numerical simulations of (1)-(3) when the lasers operate in the chaotic regime. In the simulations, we consider that the lasers are synchronized when the linear regression coefficient $r$ of the plot $I_{\mathrm{s}}(t)$ versus $I_{\mathrm{m}}(t)$ is greater than 0.9 . This is not a critical value since our simulations reveal an abrupt change of $r$ from a value around 0.7 to a value greater than 0.99 when increasing $\kappa_{c}$.

In Fig. 1, we show the values of $a$ and $\Delta \phi$ as a function of the injection coupling for several detuning values and for a value of $\kappa^{f}$ such that the dynamics is chaotic. The solid lines show the prediction of our theory given by (9) and (10) and the symbols correspond to the numerical results. Solid lines (symbols) are plotted only for the values of the coupling constant for which synchronization is theoretically (numerically) possible. When

$$
\Delta G=\Delta \gamma+\frac{2}{1+\alpha^{2}}\left[\alpha \Delta \omega+\frac{\kappa_{c}^{2}}{\gamma_{\mathrm{m}}}-\sqrt{\left(\alpha \Delta \omega+\frac{\kappa_{c}^{2}}{\gamma_{\mathrm{m}}}\right)^{2}+\left[\kappa_{c}^{2}\left(1+\frac{\Delta \gamma}{\gamma_{\mathrm{m}}}\right)-\Delta \omega^{2}\right]\left(1+\alpha^{2}\right)}\right]
$$




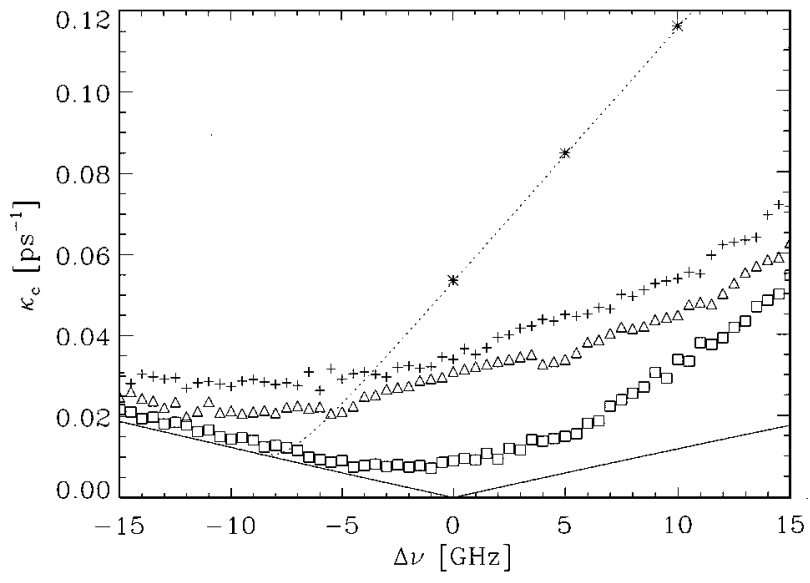

Fig. 2. Minimum and optimal coupling coefficients as function of the detuning. Solid (dotted) line corresponds to the minimum (optimal) couplings given by (12) and (13). The symbols indicate $\kappa_{c}^{\text {min }}$ obtained numerically for $\kappa^{f}=1.02 \times 10^{-2} \mathrm{ps}^{-1}$ (squares), $\kappa^{f}=1.62 \times 10^{-2} \mathrm{ps}^{-1}$ (triangles), $\kappa^{f}=2.04 \times 10^{-2} \mathrm{ps}^{-1}$ (crosses). Stars correspond to the value of $\kappa_{c}^{\text {ict }}$ obtained from numerical simulations for $\kappa^{f}=2.04 \times 10^{-2} \mathrm{ps}^{-1}$. Other parameters taken as in Fig. 1.

the lasers are synchronized, our theory gives a very accurate prediction for $a$ and $\Delta \phi$. The intersection of the solid lines/symbols in Fig. 1(a) with the horizontal line $a=1$ corresponds to the optimal coupling constant, which is precisely predicted by our theory. It can be also seen that there are frequency detunings for which the optimal coupling cannot be reached, as is the case of $\Delta \nu=-10 \mathrm{GHz}$. This value of $\Delta \nu$ is smaller than the critical one [according to (14)] that for the parameters used is $\Delta \nu_{c} \sim-8.28 \mathrm{GHz}$. For zero detuning, the phase difference is $\Delta \phi=\arctan (\alpha)$, as predicted by (9), independently of the coupling. For nonzero detuning $\Delta \phi$ approaches $\arctan (\alpha)$ as coupling increases.

The numerical results for the minimum coupling are displayed in Fig. 2 for different feedback coefficients $\kappa^{f}$. For $\kappa^{f}=1.02 \times 10^{-2} \mathrm{ps}^{-1}$, the time evolution of $I_{\mathrm{m}}$ and $I_{\mathrm{S}}$ is not fully chaotic, but quasi-periodic, while for $\kappa^{f}=1.06 \times 10^{-2} \mathrm{ps}^{-1}$ or larger it is fully chaotic. It can be seen in the figure that the minimum value of the coupling for which synchronization is numerically observed is always larger than the theoretical prediction (12), which is reasonable since the latter has been obtained as a necessary condition, rather than a sufficient one, for the existence of a synchronized solution. Furthermore, it does not tell anything about the stability of this synchronized solution. Also, the predicted value of $\kappa_{c}^{\min }$, independent of the feedback conditions, agrees better with the numerical results for low values of $\kappa^{f}$ and negative detuning. This can be understood taking into account that for large feedback, the system is more chaotic (the dimension of the chaotic attractor increases). Therefore, a larger region in the joint state space of master and slave lasers is explored, including possible unstable subsets for the synchronized solution. In fact, for $\kappa^{f}=1.02 \times 10^{-2} \mathrm{ps}^{-1}$, the time evolution of $I_{\mathrm{m}}$ and $I_{\mathrm{s}}$ is not fully chaotic but quasi-periodic. The minimum coupling is also found to increase with $\Delta \omega$ for positive detunings. The value of $\kappa_{c}^{\text {id }}$ is also displayed in the figure.
It is important to point out that for single-mode operation, the detuning can be changed continuously and the synchronization still remains. This fact is in contradiction with previous results [12], due to the fact that in [12], it was assumed that the feedback phase was the same for both lasers.

Finally, we have studied the effect of the $\alpha$ factor on the synchronization. According to (12), the minimum coupling should decrease when $\alpha$ increases. However, our prediction does not take into account the fact that as $\alpha$ increases, the dynamics is more chaotic [15]. The numerical results we obtain for, e.g., $\alpha=3$ and 5 , agree with this prediction for positive detunings and large negative ones. However, in the region of small negative detunings, the minimum coupling is smaller for $\alpha=3$. In fact, for $\alpha=3$, the laser is not chaotic, but quasi-periodic.

In conclusion, we have developed a simple theory for chaotic synchronized single-mode semiconductor lasers that accurately predicts the factor between the intensity of the ML and SL, as well as the phase difference between them. We also obtain an excellent estimation for the optimal value of the coupling coefficient and for the minimum coupling below which synchronization is not possible. The latter is always a lower bound when compared with numerical simulations.

\section{REFERENCES}

[1] K. M. Cuomo and A. V. Oppenheim, "Circuit implementation of synchronized chaos with applications to communications," Phys. Rev. Lett., vol. 71, pp. 65-68, 1993.

[2] P. Colet and R. Roy, "Digital communications with synchronized chaotic lasers," Opt. Lett., vol. 19, pp. 2056-2058, 1994.

[3] G. D. VanWiggeren and R. Roy, "Communication with chaotic lasers," Science, vol. 279, pp. 1198-1200, 1998.

[4] C. R. Mirasso, P. Colet, and P. García-Fernández, "Synchronization of chaotic semiconductor lasers: Application to encoded communications," IEEE Photon. Technol. Lett., vol. 8, pp. 299-301, 1996.

[5] V. Annovazzi-Lodi, S. Donati, and A. Scire, "Synchronization of chaotic injected-laser systems and its application to optical cryptography," IEEE J. Quantum Electron., vol. 32, pp. 953-959, June 1996.

[6] V. Ahlers, U. Parlitz, and W. Lauterborn, "Hyperchaotic dynamics and synchronization of external-cavity semiconductor lasers," Phys. Rev. E, vol. 58, pp. 7208-7213, 1998.

[7] J. P. Goedgebuer, L. Larger, and H. Porte, "Optical cryptosystem based on synchronization of hiperchaos generated by a delayed feedback tunable laser diode," Phys. Rev. Lett., vol. 80, pp. 2249-2252, 1998.

[8] S. Sivaprakasam and K. A. Shore, "Demonstration of optical synchronization of chaotic external-cavity laser diodes," Opt. Lett., vol. 24, pp. 466-468, 1999.

[9] H. Fujino and J. Ohtsubo, "Experimental synchronization of chaotic oscillations in external-cavity semiconductor lasers," Opt. Lett., vol. 25, pp. 625-627, 2000.

[10] I. Fischer, Y. Liu, and P. Davis, "Synchronization of chaotic semiconductor laser dynamics on sub-ns timescales and its potential for chaos communication," Phys. Rev. A, vol. 62, pp. 11 801(R)-11 804(R), 2000.

[11] Y. Liu, H. F. Chen, J. M. Liu, P. Davis, and T. Aida, "Synchronization of optical-feedback-induced chaos in semiconductor lasers by optical injection," Phys. Rev. A, vol. 63, pp. 031 802(R)-031 805(R), 2001.

[12] P. S. Spencer and C. R. Mirasso, "Analysis of optical chaos synchronization in frequency-detuned external cavity VCSEL's," IEEE J. Quantum Electron., vol. 35, pp. 803-809, May 1999.

[13] C. Masoller, "Anticipation in the synchronization of chaotic semiconductor lasers with optical feedback," Phys. Rev. Lett., vol. 86, pp. 2782-2785, 2001.

[14] A. Uchida, M. Shinozuka, T. Ogawa, and F. Kannari, "Experiments on chaos synchronization in two separate microchip lasers," Opt. Lett., vol. 24, pp. 890-892, 1999.

[15] G. H. M. van Tartwijk and D. Lenstra, "Semiconductor lasers with optical injection and feedback," Quantum Semiclass. Opt., vol. 7, pp. 87-143, 1995. 